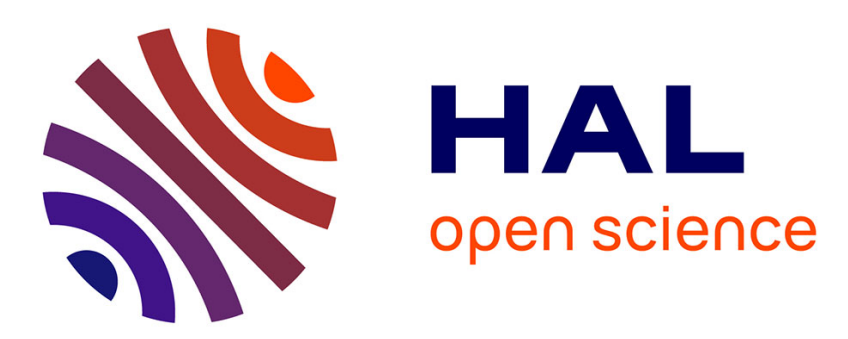

\title{
Feasibility Study of Reverse Trilateration Strategy with a single Tx for VLP
}

\author{
Chaudhary Neha, Alves Luis Nero, Zabih Ghassemlooy
}

\section{To cite this version:}

Chaudhary Neha, Alves Luis Nero, Zabih Ghassemlooy. Feasibility Study of Reverse Trilateration Strategy with a single Tx for VLP. The 2nd West Asian Colloquium on Optical Wireless Communications (WACOWC 2019), Apr 2019, Téhéran, Iran. hal-02135271

\section{HAL Id: hal-02135271 \\ https://hal.science/hal-02135271}

Submitted on 21 May 2019

HAL is a multi-disciplinary open access archive for the deposit and dissemination of scientific research documents, whether they are published or not. The documents may come from teaching and research institutions in France or abroad, or from public or private research centers.
L'archive ouverte pluridisciplinaire $\mathbf{H A L}$, est destinée au dépôt et à la diffusion de documents scientifiques de niveau recherche, publiés ou non, émanant des établissements d'enseignement et de recherche français ou étrangers, des laboratoires publics ou privés. 


\section{Feasibility Study of Reverse Trilateration Strategy with a single Tx for VLP}

\author{
Neha Chaudhary \\ Instituto de Telecomunicações \\ University of Aveiro \\ Aveiro, Portugal \\ neha.chaudhary@ua.pt
}

\author{
Luis Nero Alves \\ Instituto de Telecomunicações \\ University of Aveiro \\ Aveiro, Portugal \\ nero@ua.pt
}

\author{
Zabih Ghassemblooy \\ Optical Comm. Research Group \\ Northumbria University \\ Newcastle-upon-Tyne, UK \\ z.ghassemlooy@northumbria.ac.uk
}

\begin{abstract}
This paper describes a visible light position system relying on a single transmitter to infer position information. The system adopts a reverse trilateration scheme, where a set of three photodiodes is employed to estimate the position. The reverse trilateration scheme adopted for this system, yields to a very simple mathematical framework, suitable for low power and low complexity systems. The position information is inferred through Rx signal strength, without the need for sophisticated angle measurements or precise synchronization as is the case in angle of arrival and time difference of arrival systems. Simulation results show that the proposed system shows high susceptibility to noise, thus requiring high signal to noise ratios in order to achieve low positioning errors.
\end{abstract}

Keywords_Visible light positioning, Photodiode, RSS, Reverse Trilateration.

\section{INTRODUCTION}

The global positioning system (GPS) is an unquestionable reference when discussing positioning systems, which is widely and effectively used in outdoor environments. However, it lacks accuracy in indoor environments, mostly due to poor coverage and high signal attenuation of the radio waves propagating through solid objects. Thus, the need for a more reliable and accurate positioning technologies for indoor applications, which has seen a grown of research activity in recent years [1], [2]. Several positioning systems based on different technologies have been proposed, such as, wireless local area networks (WLAN), ultrasounds, ultra wide band (UWB), radio frequency identification (RFID) and Bluetooth. However, most of these systems have limited positioning accuracies [3-8]. System based on radio waves are affected by multipath fading [9], whereas ultrasound based systems are influenced by the ambient temperature [10]. UWB and RFID schemes have a limited range of action [11]. Finally, Bluetooth demands user association, a feature which is not always desirable [12].

Recently, visible light communications (VLC) has become an interesting and complementary communications technology to the radio frequency wireless systems [13]. Motivated by the availability of solid-state lighting devices, VLC systems explore synergies between illumination and data communications, using the same light emitting diode (LED) based lights. Visible light positioning (VLP), has emerged quite obviously as a special application of VLC, which is more suited for indoor scenarios. Given that, lighting systems come with a spatial distribution feature, using these sources as beacons (or base-stations) for positioning provides a fertile ground for research. Traditional indoor positioning techniques using LED includes, received signal strength (RSS), angle of arrival (AOA), time of arrival (TOA) and time difference of arrival (TDOA), as well as proximity and fingerprinting [14]. TOA is a well-known method for localization, which is complex requiring accurate synchronization between the transmitter (Tx) and the receiver ( $\mathrm{Rx})$ [15]. In TDOA systems the requirement for synchronization is not so stringent as for TOA, but still requires an accurate clock reference at the Tx side. AOA systems require Rx diversity in order to extract the angle information [16]. Image based systems use image sensors as a means to achieve the Rx diversity [17]. RSS systems estimate the position based on the received signal power, thus being sensitive to the noise. Nevertheless, RSS systems have higher accuracy compared to proximity and fingerprinting techniques [18], as well as being simpler and cost-effective.

One common requirement for these methods is the need to have measurements based on 3 different sources, which is a mathematical imposition, where each measurement produces a locus of possible positions. Note that, the ambiguity is removed using other measurements. Therefore, considering these constraints, a minimal set up could be achieved using a single photosensitive sensor (e.g., a photodiode (PD)), and at least three reference Txs. There have been several contributions exploring minimal configurations, which are based on different set of configurations, or combination of multiple schemes. In [19], the authors described a VLP system using two LEDs. The proposed approach carried out two RSS measurements, which were sufficient to estimate the position to the right (or left) of the line defined by the Txs. The system achieved a positioning accuracy lower $20 \mathrm{~cm}$ under moderate SNR of $13 \mathrm{~dB}$, despite having lower system complexity. In [20], a three dimensional (3D) indoor VLP system was proposed based on single $\mathrm{Tx}$ and a single tilted $\mathrm{Rx}$ as well as using RSS and AOA to improve positioning accuracy. The tilted $\mathrm{Rx}$ was mounted on a spinning platform, and the $3 \mathrm{D}$ space representation was distributed into several twodimensional (2D) planes, which were handled by lifting the Rx's platform. The proposed VLP system offered an average position error (PE) of $<35 \mathrm{~mm}$. In [21], a compact VLP system with a single $\mathrm{Tx}$ and single tilted $\mathrm{Rx}$ was proposed for an indoor environment of $0.6 \times 0.6 \times 1.1 \mathrm{~m}$ with an average $\mathrm{PE}$ of $<25 \mathrm{~mm}$. In [22], a reverse trilateration scheme employing three Rxs in the ceiling and one Tx in the ground was reported with a PE of $<0.2 \mathrm{~m}$. The proposed system was relevant for applications where the network needs to know the position of the user.

This paper focuses on the reverse trilateration scheme where the position sensor uses three PDs and relies on a single 


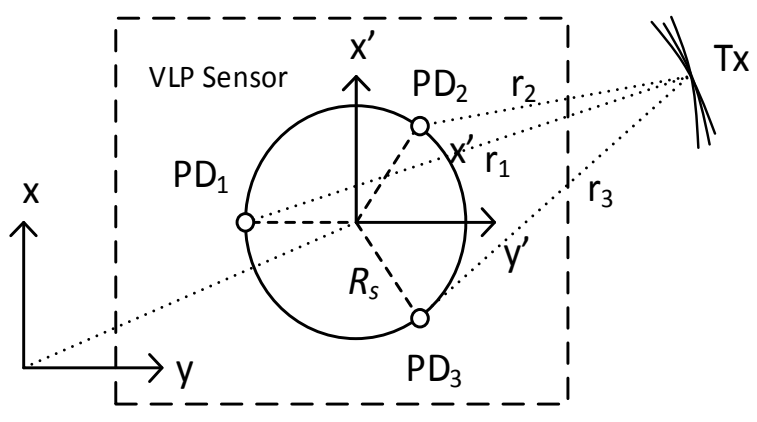

Fig.1. Reverse trilateration concept

Tx to determine the location. The system employs RSS, which makes this approach suitable for low power and low complexity applications. The paper shows theoretical analysis supported by simulation to investigate the system performance. We show that, the system's performance is highly influenced by noise. We show also, that, performance improvements can be achieved using averaging techniques.

The rest of the paper is organized as follows: Section II details the system design and modeling. Section III develops the system error performance analysis. Section IV presents the simulation set up and the achieved results. Finally section V gives the concluding remarks.

\section{SYSTEM DESIGN AND MODELING}

The schematic of the proposed reversed trilateration strategy is illustrated in Fig. 1. The VLP sensor comprises 3 PDs positioned on a circle of radius $R_{s}$. The projection of the distances between each PDs and the Tx are given by $r_{k}$, where $k=1,2$ and 3 . Using $r_{k}$ it is possible to form a well posed system of two equations for the center position of the sensor. These three distances provide the radius of three circles, centered at each PD, with common intercept at the Tx's position.

Let the positions of $k^{\text {th }} \mathrm{PD}$ be $\boldsymbol{X}^{\boldsymbol{o}}{ }_{\boldsymbol{k}}=\left(x^{o}{ }_{k}, y^{o}{ }^{o}\right)^{T}$ with $\boldsymbol{X}_{\boldsymbol{1}}=(-$ $\left.R_{S}, 0\right)^{T}, \boldsymbol{X}_{\mathbf{2}_{\mathbf{2}}}=\left(R_{S} / 2, R_{S} \sqrt{3} / 2\right)^{T}$ and $\boldsymbol{X}_{\mathbf{3}}^{\boldsymbol{o}_{3}}=\left(R_{S} / 2,-R_{S} \sqrt{ } 3 / 2\right)^{T}$. The Tx projection on the $x-y$ plane is $\boldsymbol{X}_{\boldsymbol{T}}=\left(x_{T}, y_{T}\right)^{T}$. The position of the VLP sensor in the $x-y$ plane as a function of an arbitrary translation $\boldsymbol{X}=(x, y)^{T}$ and an arbitrary rotation $\boldsymbol{R}(\theta)$ is given by:

$$
\boldsymbol{X}_{\boldsymbol{k}}=\boldsymbol{X}+\boldsymbol{R}(\theta) \boldsymbol{X}_{\boldsymbol{k}}^{o}
$$

The translation vector $\boldsymbol{X}$ is the center position of the sensor and the objective of the estimation procedure. $r_{k}$ defines a circle with the center $\boldsymbol{X}$ and passing through $\boldsymbol{X}_{\boldsymbol{T}}$, which form a set of three quadratic equations in standard form as given by:

$$
\left(\boldsymbol{X}_{T}-\boldsymbol{X}_{\boldsymbol{k}}\right)^{T}\left(\boldsymbol{X}_{T}-\boldsymbol{X}_{\boldsymbol{k}}\right)=r_{k}^{2}
$$

Note $r_{k}$ is estimated using RSS. Developing equation (2) gives:

$$
\begin{gathered}
\boldsymbol{X}_{T}^{T} \boldsymbol{X}_{T}+R_{S}^{2}+\boldsymbol{X}^{T} \boldsymbol{X}-2\left(\boldsymbol{X}_{\boldsymbol{k}}^{\boldsymbol{o}}\right)^{T} \boldsymbol{R}(\theta)^{T}\left(\boldsymbol{X}_{\boldsymbol{T}}-\boldsymbol{X}\right) \\
=r_{k}^{2}
\end{gathered}
$$

From (1) and (2) we know that $\left(\boldsymbol{X}_{\boldsymbol{k}}^{\boldsymbol{k}}\right)^{T} \boldsymbol{X}_{\boldsymbol{k}}=R_{s}{ }^{2}$ and $\boldsymbol{R}^{-1}(\theta)$ $=\boldsymbol{R}^{T}(\theta)$. The quadratic terms on the left hand-side of (3) can be removed by subtraction, thus yielding a system of two linear equations, which in the matrix form is given by:

$$
-2 R_{S} \boldsymbol{A}_{\boldsymbol{s}} \boldsymbol{R}(\theta)^{T}\left(\boldsymbol{X}_{\boldsymbol{T}}-\boldsymbol{X}\right)=\Delta \boldsymbol{r}
$$

with

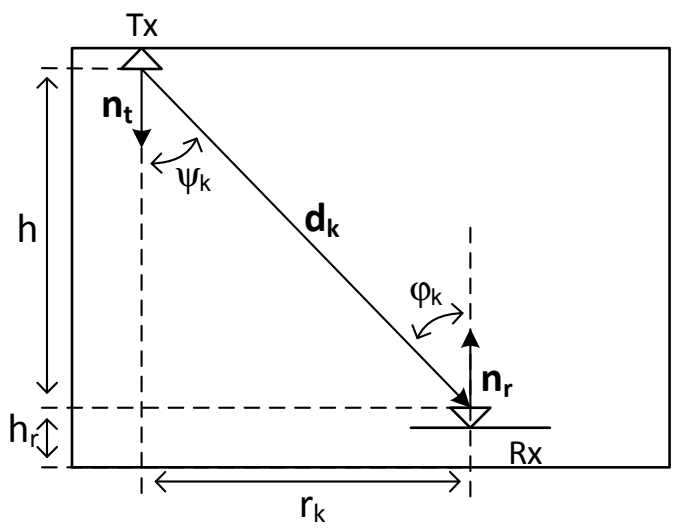

Fig. 2. System model of a single LED-based Tx and the Rx sensor with three PDs

$$
\begin{gathered}
\boldsymbol{A}_{\boldsymbol{s}}=\frac{1}{R_{s}}\left[\begin{array}{l}
\left(\boldsymbol{X}_{2}^{\boldsymbol{o}}\right)^{T}-\left(\boldsymbol{X}_{\mathbf{1}}^{\boldsymbol{o}}\right)^{T} \\
\left(\boldsymbol{X}_{3}^{\boldsymbol{o}}\right)^{T}-\left(\boldsymbol{X}_{2}^{\boldsymbol{o}}\right)^{T}
\end{array}\right]=\frac{1}{R_{s}}\left[\begin{array}{cc}
3 / 2 & \sqrt{3} / 2 \\
0 & -\sqrt{3}
\end{array}\right] \\
\Delta \boldsymbol{r}=\left[\begin{array}{l}
r_{2}^{2}-r_{1}^{2} \\
r_{3}^{2}-r_{2}^{2}
\end{array}\right]
\end{gathered}
$$

The estimated position is directly given by:

$$
\boldsymbol{X}=\boldsymbol{X}_{\boldsymbol{T}}+\frac{1}{2 R_{S}} \boldsymbol{R}(\theta) \boldsymbol{A}_{\boldsymbol{s}}^{-1} \Delta \boldsymbol{r}
$$

Equation (7) exhibits some interesting features. (i) most terms in (7) are fixed vectors and matrix except from $\Delta \boldsymbol{r}$ and $\boldsymbol{R}(\theta)$. The sensor matrix $\boldsymbol{A}_{\boldsymbol{s}}$, is fixed by the standard PD's positions, see Fig. 1 . The arbitrary rotation of the sensor can be dealt with using a compass or a gyroscope. Here, we assume that $\theta=0^{\circ}$ (i.e., no rotation). Furthermore, as it will be demonstrated, the error performance is not affected by the sensor's rotation.

\section{A The Rx Signal Strength}

Figure 2 depicts the set up conditions for signal transmission, between the Tx and the $k^{\text {th }} \mathrm{Rx}$. The objective of RSS is to measure $r_{k}$, i.e., the projection of the distance vector $\boldsymbol{d}_{\boldsymbol{k}}$ on the $x-y$ plane. These estimations are based on the DC channel gain of the communications link. The received signal for Lambertian emitter for a line of sight $(\mathrm{LoS})$ path is given by:

$$
S_{k}=H_{o} T_{s}\left(\varphi_{k}\right) g\left(\varphi_{k}\right) \frac{\cos ^{m}\left(\psi_{k}\right) \cos \left(\varphi_{k}\right)}{\left\|\boldsymbol{d}_{\boldsymbol{k}}\right\|^{2}}
$$

where

$$
\begin{aligned}
& H_{o}=\frac{m+1}{2 \pi} G_{o} \mathcal{R} A_{r} P_{t} \\
& m=-\frac{\ln (2)}{\ln (\cos (H P A))}
\end{aligned}
$$

where HPA is the half power angle for the light source, $\psi_{k}$ is the angle between $\boldsymbol{d}_{\boldsymbol{k}}$ and the LED normal, $\varphi_{k}$ is the angle between $\boldsymbol{d}_{\boldsymbol{k}}$ and the PD normal, $A_{r}$ is the PD active area, $G_{o}$ is the transimpedance gain of the $\mathrm{Rx}, \mathcal{R}$ is the $\mathrm{PD}$ responsivity and $P_{t}$ is the transmit power. $T_{s}\left(\varphi_{k}\right)$ is the $\mathrm{Rx}$ filter and $g\left(\varphi_{k}\right)$ is the optical concentrator [23]. For the forgoing analysis, we will assume that, $T_{s}\left(\varphi_{k}\right)$ and $g\left(\varphi_{k}\right)$ are both unity. Knowing that $\left\|\boldsymbol{d}_{\boldsymbol{k}}{ }^{2}\right\|=r_{k}{ }^{2}+h^{2}$ we can solve (8) to find $r_{k}^{2}$ as given by: 
The $2^{\text {nd }}$ West Asian Colloquium on Optical Wireless Communications (WACOWC2019)

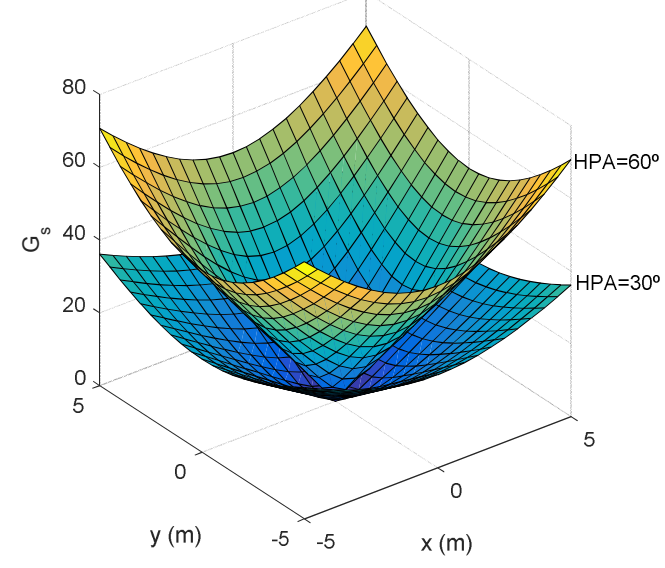

Fig. 3. The distance error geometrical factor $G_{s}$ for two different HPA values.

$$
r_{k}^{2}=\left(\frac{H_{o} h^{m+1}}{S_{k}}\right)^{\frac{2}{m+3}}-h^{2}
$$

where $h$ is the vertical distance between the Tx and the $\mathrm{Rx}$. Using (11), the $\Delta \boldsymbol{r}$ vector can be expressed as a function of the received signals on the $3 \mathrm{PDs}$ by:

$$
\begin{aligned}
& \Delta \boldsymbol{r}=\left(H_{o} h^{m+1}\right)^{\frac{2}{m+3}} \Delta_{S} \\
& \Delta_{S}=\left[\begin{array}{l}
S_{2}^{-\frac{2}{m+3}}-S_{1}^{-\frac{2}{m+3}} \\
S_{3}^{-\frac{2}{m+3}}-S_{2}^{-\frac{2}{m+3}}
\end{array}\right]
\end{aligned}
$$

\section{ERROR PERFORMANCE ANALYSIS}

The error performance analysis can be assessed using (7). The reasoning is very simple. If the detected signal is affected by noise, the measured signal power will contain errors, which are defined by small changes in $\Delta r$, i.e., $\Delta r \rightarrow \Delta r+\delta r$. Using (7) we have $\Delta \boldsymbol{X} \rightarrow \Delta \boldsymbol{X}+\delta \boldsymbol{X}$, where $\delta \boldsymbol{X}$ is the coordinate displacement due to $\delta \boldsymbol{r}$, which is given by:

$$
\boldsymbol{\delta} \boldsymbol{X}=\frac{1}{2 R_{s}} \boldsymbol{R}(\theta) \boldsymbol{A}_{\boldsymbol{s}}^{-1} \boldsymbol{\delta} \boldsymbol{r}
$$

We may transform this into a distance error, as given by:

$$
\delta X=\sqrt{\delta X^{T} \boldsymbol{\delta} X}=\frac{1}{2 R_{s}} \sqrt{\delta \boldsymbol{r}^{T} \boldsymbol{\Lambda}_{s} \boldsymbol{\delta} \boldsymbol{r}}
$$

where $\boldsymbol{\Lambda}_{\boldsymbol{s}}=\left(\boldsymbol{A}_{\boldsymbol{s}}^{-1}\right)^{T} \boldsymbol{A}_{\boldsymbol{s}}{ }^{-1}$. Equation (15) shows that, the distance error does not depend explicitly on the sensor's rotation.

\section{A The Effect of Noise on $\delta X$}

Noise can be incorporated into the error model in a very simple way. The effect of noise on the detected signal, by the $k^{\text {th }}$ PD is expressed by $S_{k} \rightarrow S_{k}+n_{k}$, where $n_{k}$ is the additive white Gaussian noise. Using the signal to noise ratio (SNR), $\gamma$, we find a simple transformation rule, which states $S_{k} \rightarrow S_{k}(1+1 / \sqrt{ } \gamma)$. Now, using (12) and (13), and re-working (15) we have:
Table.1 Default value of the system's parameters.

\begin{tabular}{ccl}
\hline & \multicolumn{2}{c}{$\mathbf{T x}-\mathbf{R x}$ parameter } \\
\hline$A_{r}$ & $100 \mathrm{~mm}^{2}$ & Area of the PD \\
$\mathcal{R}$ & $1 \mathrm{~A} / \mathrm{W}$ & Responsivity \\
$P_{t}$ & $1 \mathrm{~W}$ & Transmit power \\
$G_{o}$ & $1 \mathrm{M} \Omega$ & Rx's gain \\
$H P A$ & $60^{\circ}$ & Half power angle \\
\hline \multicolumn{3}{c}{ Geometrical Parameter } \\
\hline$d x$ & $0.5 \mathrm{~m}$ & X grid resolution \\
$d y$ & $0.5 \mathrm{~m}$ & y grid resolution \\
$\boldsymbol{n}_{\boldsymbol{t}}$ & $(0,0,-1)^{T}$ & TX heading vector \\
$\boldsymbol{n}_{\boldsymbol{r}}$ & $(0,0,1)^{T}$ & RX heading vector \\
$\boldsymbol{X}_{\boldsymbol{T}}$ & $\left(0,0, h+h_{r}\right)^{T}$ & TX position \\
$R_{S}$ & $0.1 \mathrm{~m}$ & Sensor radius \\
$h$ & $2.2 \mathrm{~m}$ & Ceiling height \\
$h_{r}$ & $0.2 \mathrm{~m}$ & Sensor height \\
$\theta$ & $0^{\circ}$ & Sensor rotation angle \\
\hline
\end{tabular}

$$
\delta X=G_{S} \frac{\left(H_{o} h^{m+1}\right)^{\frac{2}{m+3}}}{2 R_{S}}\left(\left(1+\frac{1}{\sqrt{\gamma}}\right)^{-\frac{2}{m+3}}-1\right)
$$

where $G_{s}=\left(\Delta s^{T} \Lambda_{s} \Delta s\right)^{1 / 2}$. Equation (16) exhibits the distance error dependency on all the system's parameters. It is noticeable that, the geometrical factor $G_{s}$ depends on the sensor geometry (i.e., via $\Lambda_{s}$ ) and the detected signal power. Figure 3 depicts $G_{s}$ for two different values of $H P A$ (i.e., $60^{\circ}$ and $30^{\circ}$ ), in a room of size $10 \times 10 \times 2.4 \mathrm{~m}^{3}$, with one Tx positioned in the center. As it can be seen, $G_{s}$ has a circular symmetry, showing that the distance error is indeed independent of sensor's rotation.

\section{Simulation RESUlts}

This section outlines the system set-up conditions and present the simulation results. The simulation set-up default parameter' values are given in Table 1.

\section{A General Error Performance Assessment}

The effect of noise on the estimated position can be assessed through simulation, using Matlab. This is accomplished by adopting the following steps: $(i)$ simulating the signal reception for a grid of possible Rx's positions; (ii) using (11) to calculate $r_{k}$ for each PD; (iii) estimate the position using (7); and (iv) finally, calculate the PE as the difference between the estimated and real positions. This procedure is repeated for different SNR values.

Figures 4 and 5, depicted the PE spatial distributions in a room of $10 \times 10 \times 2.4 \mathrm{~m}^{3}$, for SNRs of 50 and $60 \mathrm{~dB}$, respectively. The unusually high SNR values are a clear indication of the susceptibility of the sensor to noise. RSS relies on detected signal power, as sensor PDs are closer together, where the received signal power by the three PDs do not change much. Noise effects become of paramount importance under these circumstances. As it can be seen from Figs. 4 and 5, the error increases as we move to the periphery of the room, which is in agreement with the geometrical error dependence embedded in $G_{s}$, see Fig. 3.

Statistically speaking, it is not very relevant to compare performance in terms of extreme error achievements, since the error changes randomly for different simulations. In addition, 
The $2^{\text {nd }}$ West Asian Colloquium on Optical Wireless Communications (WACOWC2019)

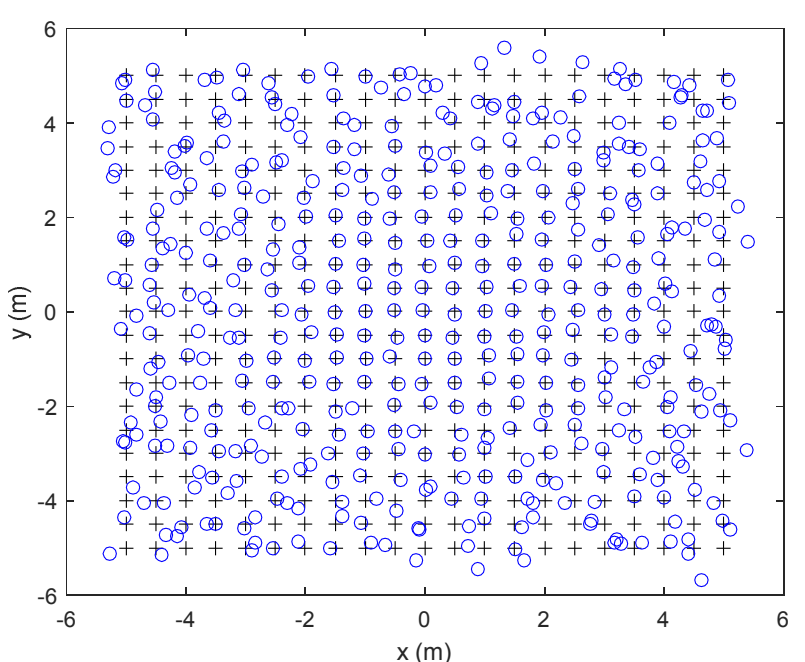

Fig. 4. The positioning error spatial distribution with $S N R=50 \mathrm{~dB}$.

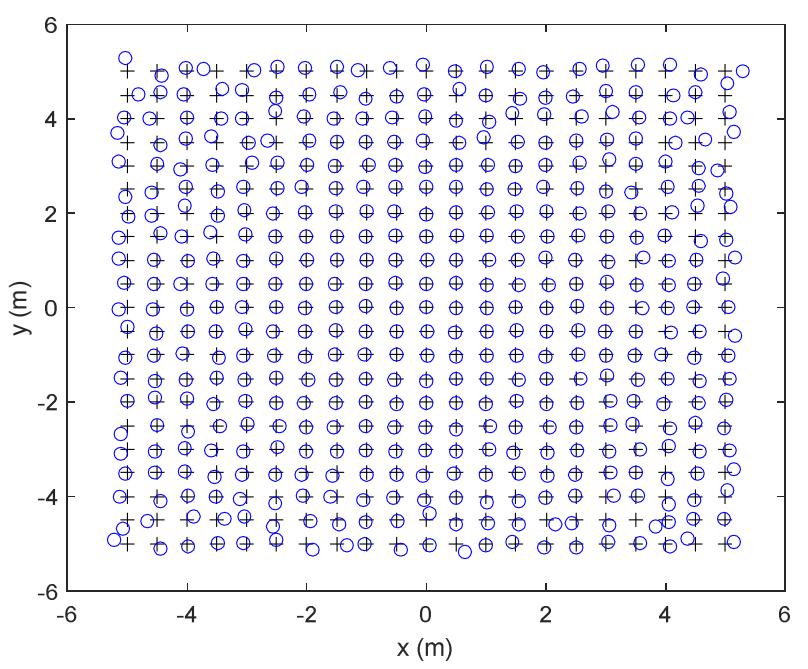

Fig. 5. The positioning error spatial distribution with $\mathrm{SNR}=60 \mathrm{~dB}$.

the average errors are not good performance indicators, as both high and low measured errors are taken into consideration with the equal weight. Instead, we propose a different error measure, which is more aligned with the probability theory. This measure, computes the percentage of estimates that fall below a certain distance criterion. In a total of $N$ estimates, let $\#\left(e_{r r}<\varepsilon\right)$ represent the number of estimates with the positioning error below $\varepsilon$, the PE is defined by:

$$
e_{r r}(\varepsilon)=\frac{\#\left(e_{r r}<\varepsilon\right)}{N}
$$

It is readily apparent that, as $N$ tends towards infinity, $e_{r r}(\varepsilon)$ tends to the probability of having error estimates below $\varepsilon$. In Figs. 4 and 5, the achieved indicators for $\varepsilon=10 \mathrm{~cm}$, were, $31.5 \%$ and $79.1 \%$ for 50 and $60 \mathrm{~dB}$, respectively.

\section{$B$ Error Performance $R_{s}$ Dependency}

Figures 6 and 7 depict the PE dependency on SNR and $R_{s}$. As before $\varepsilon=10 \mathrm{~cm}$. Fig. 6, depicts the percentage of estimates with the error below $10 \mathrm{~cm}$, for different values of $R_{s}(5,7.5$ and $10 \mathrm{~cm})$ for variable SNR values $(20$ to $70 \mathrm{~dB})$. The simulation considered a room size of $10 \times 10 \times 2.4 \mathrm{~m}^{3}$ with a grid resolution of $5 \mathrm{~cm}$ (corresponding to $N=40401$ position estimates). As it can be seen, the number of estimates with the error below $10 \mathrm{~cm}$ tends to increase with SNR, which is in agreement with (16). Lower values of $R_{s}$ require higher $S N R S$

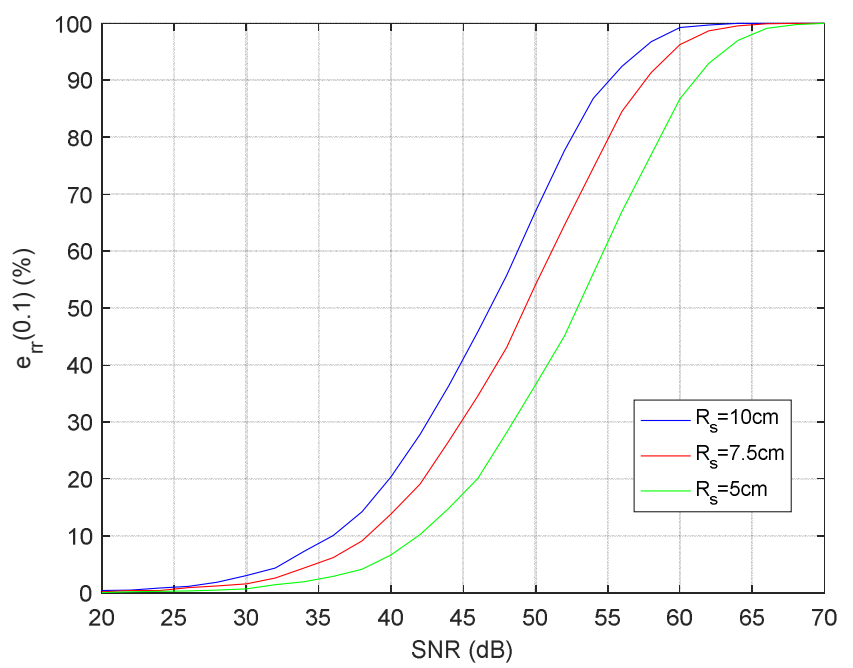

Fig. 6. The positioning error performance as function of SNR, for fixed values of $R_{S}$

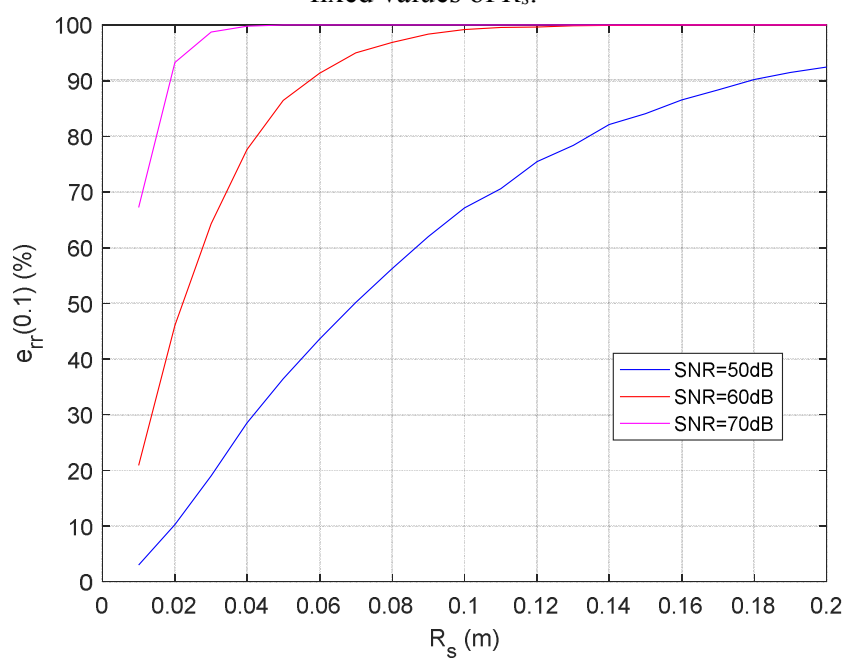

Fig. 7. The positioning error performance as function of $R_{s}$, for fixed values of SNR.

to achieve the same performance. Equation (16) seems to suggest that, the error is monotonic with $R_{s}$ - higher $R_{s}$ values mean less error. In order to further explore the error dependency on $R_{s}$, we repeated the simulation for fixed values of SNR (50, 60 and $70 \mathrm{~dB}$ ) and for range of $R_{s}$ (i.e., 1 to 20 $\mathrm{cm}$, as shown in Fig. 7. For each SNR value error performance increases monotonically with $R_{s}$. This behavior can be understood by recalling that $\Delta_{s}$ depends on the norm of the distance vectors $\boldsymbol{d}_{k}$. Since the PDs positions are function of $R_{s}$, $\boldsymbol{d}_{\boldsymbol{k}}$ depends on $R_{s}$ as well.

\section{$C$ Error Performance HPA Dependency}

Figures 8 and 9 depicts the PE dependency on both $S N R$ and $H P A$. For this study, we followed a similar approach as described in section IV-B. Figure 8 depicts the percentage of errors below $10 \mathrm{~cm}$, for fixed values of $H P A\left(45^{\circ}, 30^{\circ}\right.$ and $\left.15^{\circ}\right)$ and a range of SNR. Here too, the PE increases with the SNR and decreasing values of HPA. Note, lower HPA, means transmitting sources with higher directivity and thus higher values for $m$, which translate to less geometrical dependence $-G_{s}$ becomes flatter. Figure 9 shows the PE as a function of HPA for range of SNRs. Here we see that, the PE reduces with increasing values of $H P A$ as predicted from $G_{s}$. 
The $2^{\text {nd }}$ West Asian Colloquium on Optical Wireless Communications (WACOWC2019)

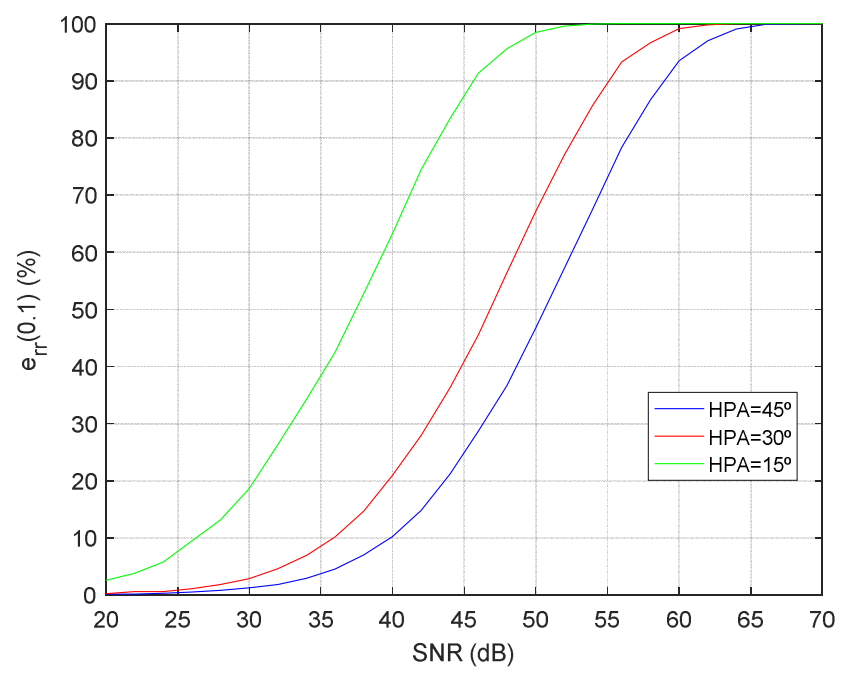

Fig. 8. The positioning error performance as function of SNR, for fixed values of HPA.

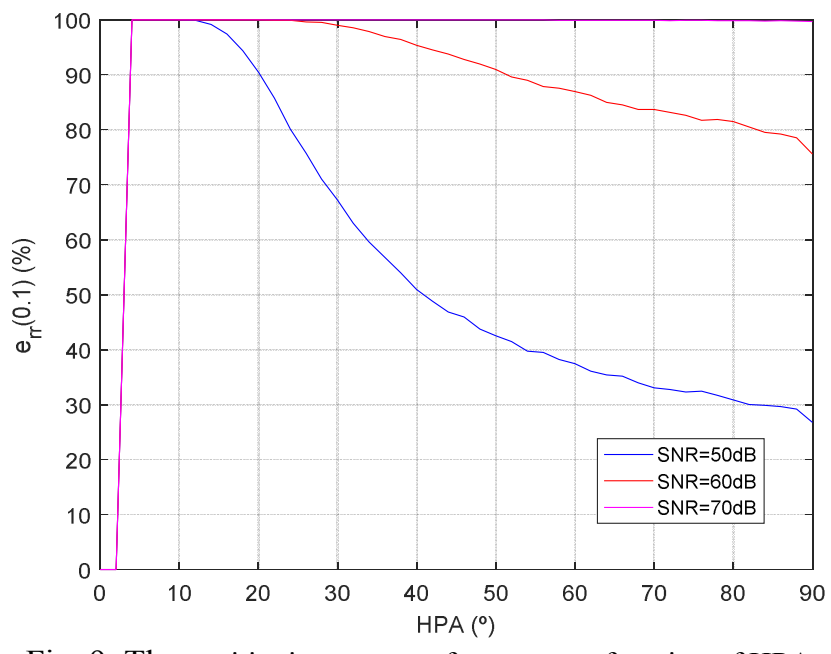

Fig. 9. The positioning error performance as function of HPA, for fixed values of SNR.

\section{$D$ Error Performance using Averaging Techniques}

Figure 10 depict the averaging effect on the PE, which shows the error below $10 \mathrm{~cm}$ for fixed values of SNR of 30, 40 and $50 \mathrm{~dB}$ as function of the number of averaging steps. It can be seen from the Fig 10 that, the error performance increases with the number of averaging steps and SNR. Therefore, it is possible to use averaging to improve PE.

\section{$\mathrm{V}$ CONCLUSIONS}

This paper presented a feasibility study on the usage of reverse trilateration for position estimation, based on RSS. The system under study, comprised a single Tx and three PDs based Rx. Simulation results disclosed that, the system is very susceptible to the noise, demanding very high SNR in order to achieve low positioning error performance. As a standalone system, the proposed scheme is not feasible. However, considering that RSS can be supported with averaging techniques (such as spread spectrum) able to improve SNR, the proposed system can represent a possible choice for low power and low complexity VLP positioning sensors. System performance analysis disclosed some important results, which could be of interest for other applications, namely: (i) the simple estimation equations can be of use to study and optimize transmitter placement in conventional trilateration schemes; and (ii) performance based on the probability of

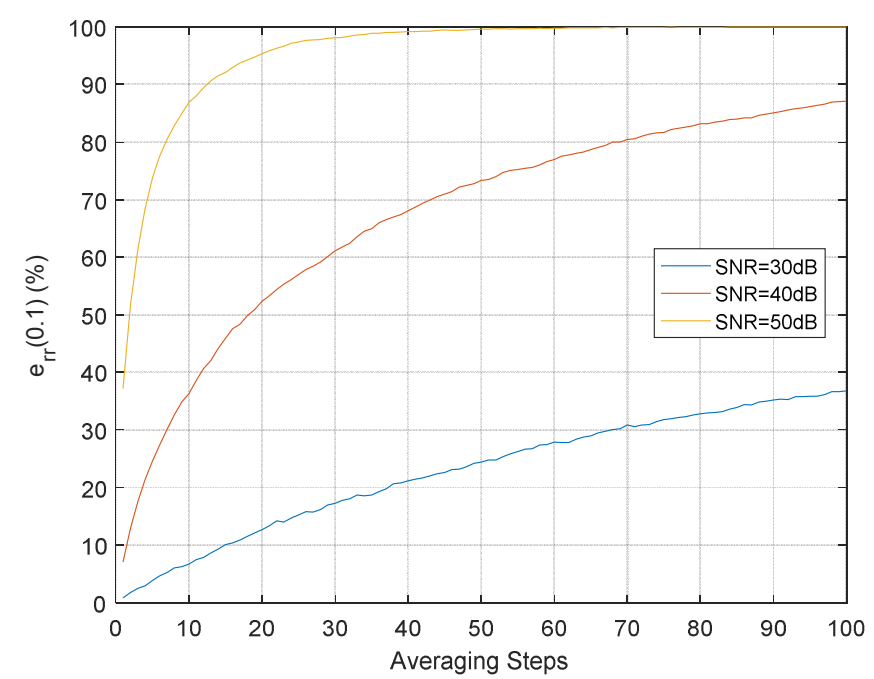

Fig. 10 - Averaging effect on the error performance for a range of SNRs.

error being lower than some predefined value can be taken as a standard approach for performance analysis in VLP systems.

\section{ACKNOWLEDGMENT}

This work is supported by H2020/MSCA-ITN funding program under the framework of European Training Network on Visible Light Based Interoperability and Networking, project (VisIoN) grant agreement no 764461.

\section{REFERENCES}

[1] W. Z . Zhang, M.I.S. Chowdhury and M. Kavehrad, "Asynchronous indoor positioning system based on visible light communication", OptEng., 53(4),2014.

[2] N. Lourenco and M. Siegel, "VLC for indoor positioning: An industrial view on applications", Ch. 12, pp. 373-404, Visible Light Communications: Theory and Applications, Eds. Z. Ghassemlooy, L N Alves, et al, CRC Press, 2017

[3] N. Priyantha, A. Miu, H. Balakrishnan, and S. Teller, "The cricket compass for context-aware mobile applications," 6th ACM Mobicom, July 2001, Rome, Italy.

[4] P. Bahl and V. N. Padmanabhan, "RADAR: An in-building RF-based user location and tracking system," in Proc. IEEE Infocom, 2000, Mar., vol. 2, pp. 775-784.

[5] S. Gezici, Z. Tian, G. B. Giannakis, H. Kobaysahi, A. F. Molisch, H. V. Poor, and Z. Sahinoglu, "Localization via ultra-wideband radios: A look at positioning aspects for future sensor networks," IEEE Signal Process. Mag., vol. 22, no. 4, pp. 77-84, Jul. 2005.

[6] D. J. Ruiz, F. S. Granja, J. C. P. Honorato, and J. I. G. Rosas, “ Accurate pedestrian indoor navigation by tightly coupling footmounted IMU and RFID measurements," IEEE Trans. Instrum. Meas., vol. 61, no. 1, pp. 178-189, Jan. 2012.

[7] Y. Po and W. Wenyan, "Efficient particle filter localization algorithm in dense passive RFID tag environment," IEEE Trans. Ind. Electron., vol. 61, no. 10, pp. 5641-5651, Oct. 2014.

[8] H. Liu, H. Darabi, P. Banerjee and J. Liu, "Survey of wireless indoor positioning techniques and systems" IEEE Transactions on Systems, Man, and Cybernetics, Part C: Applications and Reviews, 37(6):10671080, 2007.

[9] O. C. Ugweje, "Radio Frequency and Wireless Communications." The Internet Encyclopedia, April 2004. [DOI:10.1002/047148296X.tie151]

[10] B. Praher, K. Straka and G. Steinbichler, "An ultrasound-based system for temperature distribution measurements in injection moulding: system design, simulations and off-line test measurements in water", Measurement Sci. and Tech., Vol. 24, No. 8, 10 July 2013.

[11] D. Dardari, N. Decarli, A. Guerra, F. Guidi, "The future of ultrawideband localization in RFID", IEEE International Conf. on RFID, May 2016, Orlando, USA. 
[12] M. Collotta, G. Pau, T. Timothy, and Ozan K, "Bluetooth 5: a concrete step forward towards the IoT," IEEE Comm., Magaz., journ., November,2017.

[13] Z. Ghassemlooy, L. N. Alves, S. Zvanovec and M. A. Khalighi: Visible Light Communications: Theory and Applications, CRC June 2017.

[14] J. Luo, L. Fan and H. Li, "Indoor Positioning Systems Based on Visible Light Communication: State of the Art," IEEE Commun. Surveys Tuts., vol. 19, no. 4, pp. 2871-2893, 2017.

[15] D. Trong-Hop and Y. Myungsik, "TDOA-based indoor positioning using visible light," Photon . Netw. Commun., vol. 27, no. 2, pp. 8088, 2014.

[16] L. Seongsu and S. Jung, "Location awareness using angle-of-arrival based circular-pd-array for visible light communication," IEEE AsiaPacific Conference on Communications, pages 480-485, 2012.

[17] Y. Li, Z. Ghassemlooy, X. Tang, B. Lin and Y. Zhang, "A VLC Smartphone Camera based Indoor Positioning System," IEEE Photonics Technology Letters, 30 (13), pp. 1171-1174, May 2018.

[18] T. H. Do and M. Yoo, "An in-depth survey of visible light communication based positioning systems," Sensors (Basel), vol. 16, no. 5, May 2016.
[19] F. I. K. Mousa, H. L. Minh, Z. Ghassemlooy, X. Dai, S. T. Tran, A. C. Boucouvalas and S. K. Liaw, "Indoor localization system utilizing two visible light emitting diodes," Opt. Eng. 55(11), 116114, 2016.

[20] Q. L. Li, J. Y. Wang, T. Huang and Y. Wang, "Three-dimensional indoor visible light positioning system with a single Transmitter and a single tilted Receiver,” Opt. Eng. 55(10), 106103, 2016.

[21] T. Huang, X. Gao, S. Li, Q. Li, C. Li, H. Zhu and Y. Wang, "Visible light indoor positioning fashioned with a single tilted optical Rx," in Int. Conf. on Optical Communications and Networks, 2015.

[22] Y. Xu, J. Zhao, J. Shi and Nan Chi, "Reversed Three-Dimensional Visible Light Indoor Positioning Utilizing Annular Rxs with MultiPhotodiodes", MDPI journ., Sensors, August, 2016.

[23] A. Gradim, P. Fonseca, L. N. Alves and R. E. Mohamed, "On the Usage of Machine Learning Techniques to Improve Position Accuracy in Visible Light Positioning Systems," 2018 11th International Symposium on Communication Systems, Networks \& Digital Signal Processing (CSNDSP), Budapest, pp. 1-6, 2018 